\title{
The integral cohomology of the Hilbert scheme of points on a surface
}

\author{
Burt Totaro \\ UCLA Mathematics Department, Box 951555, Los Angeles, CA 90095-1555, USA; E-mail: totaro@math.ucla.edu.
}

Received: 5 January 2020; Revised: 30 June 2020; Accepted: 26 June 2020

2020 Mathematics Subject Classification: Primary-14C05; Secondary-55R80

Keywords and phrases: Hilbert scheme of points; integral cohomology; Chow motive

\begin{abstract}
We show that if $X$ is a smooth complex projective surface with torsion-free cohomology, then the Hilbert scheme $X^{[n]}$ has torsion-free cohomology for every natural number $n$. This extends earlier work by Markman on the case of Poisson surfaces. The proof uses Gholampour-Thomas's reduced obstruction theory for nested Hilbert schemes of surfaces.
\end{abstract}

\section{Contents}

1 Betti numbers of the Hilbert scheme

2 Gholampour-Thomas's reduced obstruction theory $\quad 2$

3 Torsion-freeness $\quad 3$

4 Integral Chow motive

The Hilbert scheme $X^{[n]}$ of $n$ points on a smooth complex surface $X$ is a complex manifold of dimension $2 n$ that can be viewed as a resolution of singularities of the symmetric product $S^{n} X$. The rational cohomology of $X^{[n]}$ is known, but the integral cohomology is more subtle. Any torsion in cohomology or other invariants could conceivably be useful for rationality problems.

In this paper, we show that if $X$ is a smooth complex projective surface with torsion-free cohomology, then the Hilbert scheme $X^{[n]}$ has torsion-free cohomology for every $n \geq 0$. (Since we know the Betti numbers of $X^{[n]}$ by Göttsche (stated in Theorem 1.1), this amounts to an additive calculation of $H^{*}\left(X^{[n]}, \mathbf{Z}\right)$.) We also show that if the integral Chow motive of $X$ is trivial (a finite direct sum of Tate motives), then the integral Chow motive of $X^{[n]}$ is trivial for all $n$ (Theorem 4.1).

There are some earlier results in this direction. When $X$ is the complex projective plane, Ellingsrud and Strømme found an algebraic cell decomposition of the Hilbert scheme $X^{[n]}$, which implies that its integral cohomology is torsion-free [6, Theorem 1.1]. Markman showed that the integral cohomology of the Hilbert scheme $X^{[n]}$ is torsion-free for a smooth projective surface $X$ with a nontrivial Poisson structure, or equivalently when the anticanonical bundle $-K_{X}$ has a nonzero section [11, Theorem 1]. That includes the important case where $X$ is a K3 surface, so that $X^{[n]}$ is hyperkähler. In this paper, we show that the Poisson assumption can be dropped completely. The fact that $H^{*}(X, \mathbf{Z})$ torsion-free

(C) The Author(s), 2020. Published by Cambridge University Press. This is an Open Access article, distributed under the terms of the Creative Commons Attribution licence (http://creativecommons.org/licenses/by/4.0/), which permits unrestricted re-use, distribution, and reproduction in any medium, provided the original work is properly cited. 
implies $H^{*}\left(X^{[2]}, \mathbf{Z}\right)$ torsion-free was shown (in fact, for $X$ of any dimension) in [12, Theorem 2.2]. Finally, for $X$, a smooth projective surface with first Betti number zero, Li and Qin gave an explicit basis for $H^{*}\left(X^{[n]}, \mathbf{Z}\right)$ modulo torsion [10, Theorem 1.2].

Our proofs combine Markman's ideas with the reduced obstruction theory for nested Hilbert schemes of surfaces found by Gholampour and Thomas [7].

Several related questions remain open. First, do the results of this paper extend to compact complex surfaces, or even to noncompact complex surfaces? (For the Hilbert square $X^{[2]}$, the answer is yes, by [12, Theorem 2.2].) Second, say for a smooth projective surface $X$, is the graded abelian group $H^{*}\left(X^{[n]}, \mathbf{Z}\right)$ determined by the graded abelian group $H^{*}(X, \mathbf{Z})$ when $H^{*}(X, \mathbf{Z})$ has torsion? (We know that the graded vector space $H^{*}\left(X^{[2]}, \mathbf{F}_{2}\right)$ is not determined by the graded vector space $H^{*}\left(X, \mathbf{F}_{2}\right)$, by [12, Example 2.5].) Analogously, is the integral Chow motive of $X^{[n]}$ determined by that of $X$ ? Finally, for a complex manifold $X$ of any dimension, does $H^{*}(X, \mathbf{Z})$ torsion-free imply $H^{*}\left(X^{[3]}, \mathbf{Z}\right)$ torsion-free?

\section{Betti numbers of the Hilbert scheme}

We recall here the calculation of the Betti numbers of the Hilbert schemes of points on a surface [9, equation (2.1)]. This was proved for smooth projective surfaces by Göttsche and generalized to all smooth complex analytic surfaces with finite Betti numbers by de Cataldo and Migliorini [4, Theorem 5.2.1].

Define the Poincaré polynomial of a space $Y$ by $p(Y, t)=\sum_{j} b_{j}(Y) t^{j}$.

Theorem 1.1. For a smooth complex analytic surface $X$ with finite Betti numbers, the Betti numbers of the Hilbert schemes $X^{[n]}$ are given by the generating function

$$
\sum_{n \geq 0} p\left(X^{[n]}, t\right) q^{n}=\prod_{k \geq 1} \prod_{j=0}^{4}\left(1-(-t)^{2 k-2+j} q^{k}\right)^{(-1)^{j+1} b_{j}(X)} .
$$

\section{Gholampour-Thomas's reduced obstruction theory}

Gholampour and Thomas constructed the following 'reduced' obstruction theory for nested Hilbert schemes of surfaces [7, Theorem 6.3]. This is easy when $H^{1}(X, O)=H^{2}(X, O)=0$, and in general they show how to remove the contributions of those two cohomology groups.

I would guess that the same obstruction theory exists on any complex manifold of dimension 2 . If so, then the results of this paper would extend to compact complex surfaces. Also, Gholampour and Thomas consider surfaces over the complex numbers, but their proof works verbatim over any field.

For natural numbers $n_{1} \geq n_{2}$, let $\pi$ be the projection

$$
X^{\left[n_{1}\right]} \times X^{\left[n_{2}\right]} \times X \rightarrow X^{\left[n_{1}\right]} \times X^{\left[n_{2}\right]},
$$

with the two universal subschemes $z_{1}, z_{2}$. (That is, the fiber of $z_{1}$ over a point $\left(A_{1}, A_{2}\right)$ of $X^{\left[n_{1}\right]} \times X^{\left[n_{2}\right]}$ is the 0-dimensional subscheme $A_{1}$ of $X$, and the fiber of $z_{2}$ is the 0-dimensional subscheme $A_{2}$.) Write $\mathcal{J}_{1}$ and $\mathcal{J}_{2}$ for the ideal sheaves of $z_{1}$ and $z_{2}$ on $X^{\left[n_{1}\right]} \times X^{\left[n_{2}\right]} \times X$. Finally, define

$$
R \mathcal{H} \operatorname{om}_{\pi}\left(\mathcal{J}_{1}, \mathcal{J}_{2}\right):=R \pi_{*} R \mathcal{H} \operatorname{om}\left(\mathcal{J}_{1}, \mathcal{J}_{2}\right)
$$

in the derived category of $X^{\left[n_{1}\right]} \times X^{\left[n_{2}\right]}$.

Theorem 2.1. Let $X$ be a smooth geometrically connected projective surface over a field $k$. For any $n_{1} \geq n_{2}$, the 2-step nested Hilbert scheme $X^{\left[n_{1}, n_{2}\right]}$ (of 0-dimensional subschemes of degree $n_{1}$ containing a subscheme of degree $n_{2}$ ) carries a natural perfect obstruction theory whose virtual cycle

$$
\left[X^{\left[n_{1}, n_{2}\right]}\right]^{\mathrm{vir}} \in C H_{n_{1}+n_{2}}\left(X^{\left[n_{1}, n_{2}\right]}\right)
$$


has pushforward to the Chow groups of $X^{\left[n_{1}\right]} \times X^{\left[n_{2}\right]}$ equal to the Chern class $c_{n_{1}+n_{2}}\left(R \mathcal{H o m} \operatorname{fom}_{\pi}\left(\mathcal{J}_{1}, \mathcal{J}_{2}\right)[1]\right)$.

We only need the case $n_{1}=n_{2}$ of Theorem 2.1. That is:

Corollary 2.2. Let $X$ be a smooth geometrically connected projective surface over a field $k$. Then the Hilbert scheme $X^{[n]}$ carries a natural perfect obstruction theory whose virtual cycle

$$
\left[X^{[n]}\right]^{\mathrm{vir}} \in \mathrm{CH}_{2 n}\left(X^{[n]}\right)
$$

has pushforward by the diagonal morphism to $X^{[n]} \times X^{[n]}$ equal to the Chern class $c_{2 n}\left(R \mathcal{H} \operatorname{lom}_{\pi}\left(\mathcal{J}_{1}, \mathcal{J}_{2}\right)[1]\right)$.

Here $\mathrm{CH}_{2 n}\left(X^{[n]}\right)$ is $\mathbf{Z}$ times the class of $X^{[n]}$, and it follows from Gholampour-Thomas's construction that the class of the virtual cycle in Corollary 2.2 is the integer 1 times the class of $X^{[n]}$. Namely, the perfect obstruction theory on $X^{\left[n_{1}, n_{2}\right]}$ in Theorem 2.1 can be written as

$$
\left\{\left.T\left(X^{\left[n_{1}\right]} \times X^{\left[n_{2}\right]}\right)\right|_{X^{\left[n_{1}, n_{2}\right]}} \rightarrow \mathcal{E} x t_{p}^{1}\left(\mathcal{J}_{1}, \mathcal{J}_{2}\right)_{0}\right\}^{\vee} \rightarrow L_{X^{\left[n_{1}, n_{2}\right]}}
$$

in the derived category of $X^{\left[n_{1}, n_{2}\right]}$ [7, Corollary 6.33]. Here $L_{Y}$ denotes the cotangent complex of $Y$, and $p$ denotes the projection $X^{\left[n_{1}, n_{2}\right]} \times X \rightarrow X^{\left[n_{1}, n_{2}\right]}$. Since $\mathcal{J}_{1}$ and $\mathcal{J}_{2}$ are flat over $X^{\left[n_{1}\right]} \times X^{\left[n_{2}\right]}$, they restrict to ideal sheaves on $X^{\left[n_{1}, n_{2}\right]} \times X$, which we also call $\mathcal{J}_{1}$ and $\mathcal{J}_{2}$. At a point $\left(I_{1}, I_{2}\right)$ in $X^{\left[n_{1}, n_{2}\right]}$, we define

$$
\mathcal{E} x t_{p}^{1}\left(\mathcal{J}_{1}, \mathcal{J}_{2}\right)_{0}=\operatorname{coker}\left(H^{1}(X, O) \rightarrow \operatorname{Ext}_{X}^{1}\left(I_{1}, I_{2}\right)\right),
$$

where that map is associated with the given inclusion $I_{1} \rightarrow I_{2}$.

Here, $\mathcal{E} x t_{p}^{1}\left(\mathcal{J}_{1}, \mathcal{J}_{2}\right)_{0}$ is the tangent sheaf to $X^{\left[n_{1}, n_{2}\right]}$. Therefore, the perfect obstruction theory on $X^{[n]}$ in Corollary 2.2 is

$$
\left\{T X^{[n]} \oplus T X^{[n]} \rightarrow \mathcal{E} x t_{p}^{1}\left(\mathcal{J}_{1}, \mathcal{J}_{2}\right)_{0}\right\}^{\vee} \rightarrow L_{X^{[n]}}
$$

In this case, $\mathcal{J}_{1}$ and $\mathcal{J}_{2}$ are the same, and the map is the sum of two isomorphisms $T X^{[n]} \rightarrow \mathcal{E} x t_{p}^{1}(\mathcal{J}, \mathcal{J})_{0}$. So this perfect obstruction theory is equivalent to the obvious one on the smooth variety $X^{[n]}$, and so the resulting virtual cycle is 1 times the fundamental class of $X^{[n]}$.

\section{Torsion-freeness}

Theorem 3.1. Let $X$ be a smooth complex projective surface. If $H^{*}(X, \mathbf{Z})$ is torsion-free, then $H^{*}\left(X^{[n]}, \mathbf{Z}\right)$ is torsion-free for every $n \geq 0$.

More generally, for any prime number $p$, the same proof works $p$-locally. That is, if $H^{*}(X, \mathbf{Z})$ has no $p$-torsion, then $H^{*}\left(X^{[n]}, \mathbf{Z}\right)$ has no $p$-torsion for every $n \geq 0$.

Proof. We follow Markman's argument on Poisson surfaces, with the extra input of Corollary 2.2 [11, proof of Theorem 1]. Bott periodicity says that topological $K$-theory is 2-periodic. The differentials in the Atiyah-Hirzebruch spectral sequence from $H^{*}(X, \mathbf{Z})$ to $K^{*}(X)$ are always torsion [2, Section 2.4]. Since $H^{*}(X, \mathbf{Z})$ is torsion-free, the spectral sequence degenerates at the $E_{2}$ page. Also, the abelian group $H^{*}(X, \mathbf{Z})$ is finitely generated because $X$ is a closed manifold. Therefore, $K^{*}(X)$ is a finitely generated free abelian group, with $K^{0}(X)$ of rank $b_{2}(X)+2$ and $K^{1}(X)$ of rank $2 b_{1}(X)$. In this situation, the Künneth formula holds for $K$-theory:

$$
K^{0}(X \times Y) \cong\left[K^{0}(X) \otimes_{\mathbf{Z}} K^{0}(Y)\right] \oplus\left[K^{1}(X) \otimes_{\mathbf{Z}} K^{1}(Y)\right]
$$

for every finite CW-complex $Y$ [1, Corollary 2.7.15]. 
Let $\left\{x_{1}, \ldots, x_{m}\right\}$ be a homogeneous basis for $K^{0}(X) \oplus K^{1}(X)$. Write $u \mapsto u^{\vee}$ for the involution on $K^{0}$ of a space that takes a vector bundle to its dual, also known as the Adams operation $\psi^{-1}$. (For a coherent sheaf $E$ on a smooth scheme $Y$, we interpret $E^{\vee}$ to mean $\operatorname{RHom}\left(E, O_{Y}\right)$ in the derived category of $Y$, so it defines the same operation on $K^{0}(Y)$.) Consider the Künneth decomposition

$$
\mathcal{J}=\sum_{i=1}^{m} x_{i} \otimes e_{i}
$$

of the class of the universal ideal sheaf $\mathcal{J}$ in $K^{0}\left(X \times X^{[n]}\right)$. Here, the $e_{i}$ are some (homogeneous) elements of $K^{*}\left(X^{[n]}\right)$. Likewise, write

$$
(\mathcal{J})^{\vee}=\sum_{i=1}^{m} e_{i}^{\prime} \otimes x_{i}
$$

in $K^{0}\left(X^{[n]} \times X\right)$ for some (homogeneous) elements $e_{i}^{\prime} \in K^{*}\left(X^{[n]}\right)$. Write $\chi: K^{*}(X) \rightarrow \mathbf{Z}$ for pushforward to a point (which is defined because $X$ is a compact complex manifold). For a coherent sheaf $E$, this is given by $\chi(E)=\sum_{j}(-1)^{j} h^{j}(X, E)$.

Write $\pi_{i j}$ for the projection from $X^{[n]} \times X \times X^{[n]}$ to the product of the $i$ th and $j$ th factors. Then we have the equality in $K^{0}\left(X^{[n]} \times X^{[n]}\right)$ :

$$
\left(\pi_{13}\right)_{*}\left[\pi_{12}^{*}(\mathcal{J})^{\vee} \otimes^{L} \pi_{23}^{*}(\mathcal{J})\right]=\sum_{i=1}^{m} \sum_{j=1}^{m}\left(\pi_{13}\right)_{*}\left(e_{i}^{\prime} \otimes\left(x_{i} x_{j}\right) \otimes e_{j}\right) .
$$

For $x, y \in K^{*}(X)$, define $(x, y)=-\chi(x y) \in \mathbf{Z}$, the sign being conventional for the Mukai pairing. Using the projection formula, we have

$$
\left(\pi_{13}\right)_{*}\left[\pi_{12}^{*}(\mathcal{J})^{\vee} \otimes^{L} \pi_{23}^{*}(\mathcal{J})\right]=-\sum_{i=1}^{m} \sum_{j=1}^{m}\left(x_{i}, x_{j}\right) e_{i}^{\prime} \otimes e_{j} .
$$

We need Markman's definition of the Chern classes of an element of $K^{1}(Y)$, say for a finite CW complex $Y$ [11, Definition 19]. First, identify $K^{1}(Y)$ with $\widetilde{K}^{0}\left(\Sigma Y^{+}\right)$, where $Y^{+}$means the union of $Y$ with a disjoint base point, and $\widetilde{K}$ is the reduced $K$-theory of a pointed space. For $u \in K^{1}(Y)$ and $i \geq 1 / 2$ congruent to $1 / 2$ modulo $\mathbf{Z}$, define the Chern class $c_{i}(u)$ as the image in $H^{2 i}(Y, \mathbf{Z})$ of $c_{i+1 / 2}(\widetilde{u})$, where $\widetilde{u}$ is the corresponding element of $\widetilde{K}^{0}\left(\Sigma Y^{+}\right)$, and we identify $H^{2 i}(Y, \mathbf{Z})$ with $\widetilde{H}^{2 i+1}\left(\Sigma Y^{+}, \mathbf{Z}\right)$. For $u, v \in K^{1}(Y)$, Markman showed that the Chern classes of $u v \in K^{0}(Y)$ can be written as polynomials with integer coefficients in the even-dimensional classes $c_{i}(u) c_{j}(v)$ [11, Lemma 21].

By Corollary 2.2, it follows that the diagonal $\Delta \in H^{4 n}\left(X^{[n]} \times X^{[n]}, \mathbf{Z}\right)$ is given by

$$
\Delta=c_{2 n}\left(\sum_{i=1}^{m} \sum_{j=1}^{m}\left(x_{i}, x_{j}\right) e_{i}^{\prime} \otimes e_{j}\right) .
$$

By the formulas for the Chern classes of direct sums and tensor products of elements of $K^{0}$, together with the result above on Chern classes of the product of two elements of $K^{1}$, it follows that $\Delta$ can be expressed as a sum

$$
\Delta=\sum_{j \in J} \alpha_{j} \otimes \beta_{j}
$$

where each $\alpha_{j}$ and $\beta_{j}$ is a polynomial with integer coefficients in the Chern classes of $e_{1}, \ldots, e_{m}, e_{1}^{\prime}, \ldots, e_{m}^{\prime}$. 
Viewed as a correspondence, the diagonal acts as the identity on integral cohomology. That is, for any element $u \in H^{*}\left(X^{[n]}, \mathbf{Z}\right)$, we have

$$
u=\left(p_{1}\right)_{*}\left(\Delta \cdot p_{2}^{*}(u)\right) .
$$

Combining this with the decomposition of the diagonal above, we find that $u$ is a $\mathbf{Z}$-linear combination of the elements $\alpha_{j}$ :

$$
u=\sum_{j \in J}\left(\int_{X^{[n]}} u \beta_{j}\right) \alpha_{j} .
$$

If $u$ is torsion, then all the intersection numbers $\int u \beta_{j} \in \mathbf{Z}$ are zero, and so $u=0$. That is, $H^{*}\left(X^{[n]}, \mathbf{Z}\right)$ is torsion-free, as we want.

\section{Integral Chow motive}

Finally, we show that if the Chow motive with integral coefficients of a smooth projective surface $X$ over a field $k$ is trivial (a direct sum of Tate motives), then the same holds for all Hilbert schemes $X^{[n]}$. The analogous statement with rational coefficients is known, by de Cataldo and Migliorini's general description of the motive of $X^{[n]}$ with rational coefficients [5, Theorem 6.2.1].

The Chow motive with integral coefficients is a direct sum of Tate motives for every smooth complex projective rational surface, but also for some Barlow surfaces, which are of general type [3, Proposition 1.9], [13, Theorem 4.1].

Theorem 4.1. Let $X$ be a smooth projective surface over a field $k$. Let $R$ be a PID of characteristic zero, meaning that $\mathbf{Z}$ is a subring of $R$. If the Chow motive of $X$ with coefficients in $R$ is a finite direct sum of Tate motives $R(a)$, then the Hilbert scheme $X^{[n]}$ has the same property for every $n \geq 0$.

Proof. By Gorchinsky and Orlov, since the Chow motive of $X$ with coefficients in $R$ is a finite direct sum of Tate motives and $\mathbf{Z}$ is a subring of $R$, the $K$-motive of $X$ with coefficients in $R$ is a finite direct sum of $K$-motives of points [8, Proposition 4.1]. It follows that the Künneth formula holds for algebraic $K$-theory of products with $X$, meaning that for every smooth projective variety $Y$, the product map

$$
K_{0}(X) \otimes_{\mathbf{Z}} K_{0}(Y) \otimes_{\mathbf{Z}} R \rightarrow K_{0}(X \times Y) \otimes_{\mathbf{Z}} R
$$

is an isomorphism.

Given that, the proof of Theorem 3.1 produces elements $e_{i}, e_{i}^{\prime}$ in $K_{0}\left(X^{[n]}\right) \otimes R$ using the Künneth formula on $X \times X^{[n]}$. The argument then shows that the diagonal in the Chow group $C H^{2 n}\left(X^{[n]} \times\right.$ $\left.X^{[n]}\right) \otimes R$ is completely decomposable as a sum $\sum_{j} \alpha_{j} \otimes \beta_{j}$. Using that $R$ is a PID, it follows that the Chow motive of $X^{[n]}$ with coefficients in $R$ is a finite direct sum of Tate motives $R(a)$ [13, proof of Theorem 4.1].

Acknowledgements. I thank Stefan Schreieder for useful discussions. This work was supported by NSF grant DMS-1701237.

Conflict of Interest: None.

\section{References}

[1] M. Atiyah, K-Theory (W. A. Benjamin, New York, 1967).

[2] M. Atiyah and F. Hirzebruch, 'Vector bundles and homogeneous spaces', in Proc. Sympos. Pure Math., vol. 3 (American Mathematical Society, 1961), 7-38.

[3] A. Auel, J.-L. Colliot-Thélène, and R. Parimala, 'Universal unramified cohomology of cubic fourfolds containing a plane', in Brauer Groups and Obstruction Problems (Palo Alto, 2013) (Birkhäuser, 2017), 29-56.

[4] M. A. de Cataldo and L. Migliorini, 'The Douady space of a complex surface', Adv. Math. 151 (2000), 283-312. 
[5] M. A. de Cataldo and L. Migliorini, 'The Chow groups and the motive of the Hilbert scheme of points on a surface', J. Alg. 251 (2002), 824-848.

[6] G. Ellingsrud and S. Strømme, 'On the homology of the Hilbert scheme of points in the plane', Invent. Math. 87 (1987), $343-352$.

[7] A. Gholampour and R. P. Thomas, 'Degeneracy loci, virtual cycles and nested Hilbert schemes. I', Tunisian J. Math. 2 (2020), 633-665.

[8] S. Gorchinskiy and D. Orlov, 'Geometric phantom categories', Publ. Math. IHES 117 (2013), 329-349.

[9] L. Göttsche, 'Hilbert schemes of points on surfaces', in Proceedings of the International Congress of Mathematicians (Beijing, 2002), vol. 2 (Higher Education Press, Beijing, 2002), 483-494.

[10] Wei-Ping Li and Zhenbo Qin, 'Integral cohomology of Hilbert schemes of points on surfaces', Comm. Anal. Geom. 16 (2008), 969-988.

[11] E. Markman, 'Integral generators for the cohomology ring of moduli spaces of sheaves over Poisson surfaces', Adv. Math. 208 (2007), 622-646.

[12] B. Totaro, 'The integral cohomology of the Hilbert scheme of two points', Forum Math. Sigma 4 (2016), e8, 20 pp.

[13] B. Totaro, 'The motive of a classifying space', Geometry and Topology 20-4 (2016), 2079-2133. 